\title{
On the Choice of Norm for Modeling Compressible Flow Dynamics at Reduced-Order using the POD $^{1}$
}

\author{
Tim Colonius ${ }^{2}$ and Clancy W. Rowley ${ }^{3}$ and Jonathan B. Freund ${ }^{4}$ and Richard M. Murray ${ }^{5}$
}

\begin{abstract}
We use POD/Galerkin projection to investigate and derive reduced-order models of the dynamics of compressible flows. We examine DNS data for two flows, a turbulent $M=0.9$ jet and self-sustained oscillations in the flow over an open cavity, and show how different choices of norm lead to different definitions of the energetic structures, and, for the cavity, to different reduced-order models of the dynamics. For the jet, we show that the near-field dynamics are fairly well represented by relatively few modes, but that key processes of interest, such a acoustic radiation, are not well captured by norms that are defined based on volume integrals of pressure and velocity. For the cavity flow, we demonstrate that vector-valued POD modes lead to reduced-order models that are much more effective (accurate and stable) than scalar-valued modes defined independently for different flow variables.
\end{abstract}

\section{Introduction}

The Proper Orthogonal Decomposition (POD) $[6]$ is an attractive means of using data to generate an optimal set of basis functions that represent the "energy" of the data, defined by a user-selected norm. This basis is optimal in the sense that a finite number of these orthogonal modes represent more of the energy than any other set of orthogonal modes. POD modes can be useful in modeling the dynamics of the flow, either by the 'inspiration' they provide in highlighting dynamically significant structure in the data, or quantitatively by Galerkin projection of a relatively small number of modes onto the governing equations to generate a reduced-order model. If the reduced-order model faithfully represents the dynamical process in a flow leading to a particular end-product (examples being drag on a body or sound generated by turbulence), they may be used as a basis for a control design that attempts to alter the end-product, such as reducing drag or radiated

\footnotetext{
${ }^{1}$ Supported in part by AFOSR F49620-98-1-0095 (TC) and NASA (JBF)

${ }^{2}$ California Institute of Technology (coloniusecaltech.edu)

${ }^{3}$ Princeton University (cwrovley@princeton.edu)

${ }^{4}$ University of Illinois at Urbana-Champaign (jbfreundQuiuc.edu)

${ }^{5}$ California Institute of Technology (murray@caltech.edu)
}

sound.

There are, however, some obstacles to achieving this goal in practice. For compressible flows, one must first decide on an appropriate generalization of previous concepts developed for incompressible flows. Generally, POD modes can be defined for any vector of flow quantities, in any well-defined, weighted norm. We shall show that different choices of flow quantities will highlight different relations between the variables. A theme of our work, therefore, has been to study how the choice of norm dictates the structure of the POD modes, the extent to which they represent particular quantities (aside from the norm for which they were designed), and, ultimately, the efficacy of a reducedorder models based on Galerkin projection of equations of motion onto the POD basis.

Our primary application is to obtain reduced-order models for sound generation by large-scale structures in turbulent flows. We consider here two examples, selfsustaining flow/acoustic oscillations in the flow over an open cavity, and sound generation by a turbulent transonic jet. The sound radiated by turbulence is a very small fraction of the total energy, and norms that highlight the near-field energetic structures may not be an efficient way to represent the noise generating flow, even if the dynamics of these structures are ultimately responsible for generating the sound. An intriguing question is whether an appropriate norm can be defined that would efficiently represent the sound producing dynamics of the flow, and a long term goal of the present work is to address this issue. In other words, we seek to determine what is a good norm to define such that relatively few modes contribute to the generation of radiated sound. This, we hope, will in turn lead to phenomenological models for sound radiation by large scale structures in turbulent jets and other flows.

\section{POD modes for Compressible Flow}

We seek a representation for a particular flow quantity, or a vector of flow quantities, functions of space and time, $\mathrm{q}(x, t)$, as an expansion in scalar or vectorvalued orthogonal modes, $\varphi_{j}(x)$. The POD expansion provides an optimal convergent series representation of 
a specified $L_{2}$ norm of $\mathbf{q}$ than any other expansion. For incompressible flow fields, this norm is typically taken to be the fluctuation kinetic energy, and therefore $\mathbf{q}=(u, v, w)$, where $u, v$, and $w$ are the three velocity components (in an appropriately defined coordinate system), and the norm is defined over the entire volume of flow. In general, however, we can quite arbitrarily specify $q$, the region over which the norm is defined, and how the individual components of $\mathbf{q}$ are weighted in the norm.

For compressible flows, the best choices of variables, norms, and weightings are not obvious. Some first attempts at performing POD for these flows used more than one norm-one for each relevent flow variable, and we refer to the modes so-determined as scalar-valued modes. The only connections between flow variables in these cases is that they are computed with the same snapshots from the overall flow solutions. However, there are interrelations between the various variables in a compressible flow. For example, the divergence of the velocity is closely related, through the continuity equation, to the density (and through the equation of state, in turn, to other thermodynamic variables). It would seem that an appropriate vector-valued norm will better preserve such relations. One possibility is to use a vector of, say, primative variables (density, velocity, pressure) or conserved quantities (density, momenta, total energy), but there is no physical interpretation of the norm in that case (even if nondimensional versions are used so as to avoid, say, adding densities and velocities). Moreover, the general compressible NavierStokes equations (plus continuity and energy) then involve division by the density and this complicates the Galerkin project of the equations onto the POD modes.

Rowley et al[9] found that a norm based on the stagnation enthalpy was particularly useful, wherein $\mathbf{q}=$ $(u, v, w, a)$, where $a$ the speed of sound. The stagnation enthalpy was then

$$
\|\mathbf{q}\|^{2}=\int_{\Omega} \frac{2 a^{2}}{\gamma-1}+v_{x}^{2}+v_{r}^{2}+v_{\theta}^{2} d V
$$

where again, $\Omega$ is the region of interest. For flows that are isentropic (or nearly so), this definition yields equations of motion with only quadratic nonlinearities and results in a particularly simple Galerkin projection. This definition is used in the development of reducedorder models for cavity oscillations discussed below.

In constructing POD modes for turbulent jets, we have defined a more general set of variables that include pressure. The principal reason for this was to compare with earlier studies [1] where modes defined on the pressure alone were used. So we generalize the above norm and use $\mathbf{q}=(u, v, w, a, p)$ with scaling fac- tor $\alpha=\left(\alpha_{1}, \ldots, \alpha_{5}\right)$ and define

$$
\|\mathbf{q}\|^{2}=\int_{\Omega} \alpha_{1} v_{x}^{2}+\alpha_{2} v_{r}^{2}+\alpha_{3} v_{\theta}^{2}+\alpha_{4} a^{2}+\alpha_{5} p^{2} d V,
$$

where a consistent non-dimensionalization of $q$ is implied. The constants $\alpha$ determine the specific norm. Choosing $\boldsymbol{\alpha}=\left(1,1,1, \frac{2}{\gamma-1}, 0\right)$ recovers the stagnation enthalpy norm used by Rowley, [9] and $\alpha=(1,1,1,0,0)$ recovers the standard kinetic energy norm used in incompressible flow.

Finally, it is well known that for homogeneous (periodic) coordinate directions, Fourier modes are identical to POD modes. In the jet flow we describe below, we anticipate this result by starting with the azimuthal Fourier transform of $q$,

$$
\mathbf{q}(x, r, t)=\overline{\mathbf{q}}(x, r)+\sum_{m=1}^{M} \mathbf{q}^{m}(x, r, t) e^{i m \theta},
$$

where we have removed the mean, $\overline{\mathbf{q}}$, and compute POD modes for each $m$ as

$$
\mathbf{q}^{m}(x, r, t)=\sum_{j=1}^{N} a_{j}^{m}(t) \varphi_{j}^{m}(x, r) .
$$

In the examples considered below, we use data from $\mathrm{Di}-$ rect Numerical Simulation (DNS) of the full unsteady compressible Navier-Stokes equations in two and three dimensions. Computational methods and validations are described in some previous papers $[10,5]$. We compute POD modes using the method of snapshots[12] from simulation data saved at discrete times.

\section{Reduced-order models of cavity flow/acoustic oscillations}

Self-sustained oscillations over open cavities are thought to arise from a naturally occurring feedback loop[8]. Small flow disturbances are amplified by the free shear layer spanning the cavity, scattered into acoustic waves at the cavity trailing edge, propagate upstream, and excite further disturbances in the shear layer. Recently there has been renewed interest in attenuating these oscillations by means of active control. These efforts have involved open-loop control[13], or phase-locked loops, designed to cancel flow disturbances at a particular frequency $[7,2,11,14,4]$. None of the control strategies attempted so far have used an explicit dynamical model for control design, or analysis of performance or robustness. In many cases, even a simple low-dimensional model can capture important features of a system, and both indicate effective control strategies, and reveal limitations of active control. 
We report here reduced-order models based on the POD/Galerkin approach using data from twodimensional DNS. A detailed study of the physics of cavity oscillations, based on the DNS, is presented in a recent paper[10]. The data used for the reduced-order models presented here are from a run with $L / D=2$, where $L$ and $D$ are the cavity length and depth, a freestream Mach number of $0.6, L / \theta=58.4$, where $\theta$ is the momentum thickness of the boundary layer at the leading edge of the cavity, and a Reynolds number based on $\theta$ of 68.5. We use the (two-dimensional) volume based norm based on the primitive variables independently (scalar modes for $u, v, \rho$, and $p$ ) as well as the vector norm based on $\left(\alpha=\left(1,1,1, \frac{2}{\gamma-1}, 0\right)\right)$. The volume is defined as a portion of the (2D) DNS domain near the cavity (see ref.[9] for details). We obtain an approximate version of the Navier-Stokes equations, valid for cold flows at moderate Mach number, and project these equations onto the POD basis. The approximate equations involve only quadratic nonlinearities, and the resulting projections are much simpler than those based on the full compressible Navier-Stokes equations [9].

By contrast with the fully turbulent jet flow discussed below, the two-dimensional cavity flow is transitional. We compute POD modes based on snapshots of the developing flow (transient portion of the simulation where the self-sustaining oscillations are being established), as well as the fully-developed limit-cycle flow. As we might expect, the latter is well represented by just a few modes; we find in this case that modes come in pairs, and seem to represent constant frequency oscillations (like sine and cosine). For the vector-valued enthalpy modes, the first six modes capture $99.6 \%$ of the energy for the fully-developed flow. The structure of the modes (figure 1), depicted in terms of curl (vorticity) and divergence of velocity (acoustic) fluctuations, shows clearly the instability wave spanning the cavity shear layer (vorticity) and the acoustic coupling produced by radiation near the trailing edge (divergence).

The behavior of reduced-order models, based on Galerkin project onto the POD basis are depicted in figure 2, for both scalar and vector-valued modes. Note that in the scalar case, a " 4 mode" model requires 4 modes for each flow variable $(u, v, h)$, resulting in 12 modes altogether, and 12 ODEs to be solved, whereas in the vector case, a " 4 mode" model requires exactly 4 ODEs to be solved. For all cases, the initial condition is obtained by projecting a snapshot from the DNS (the first snapshot used for the POD computation) onto the POD modes.

All of the scalar-valued models are accurate for short time, but deviate significantly for longer time. Furthermore, taking more modes does not always help: for long times, the most accurate model is the 2-mode model!

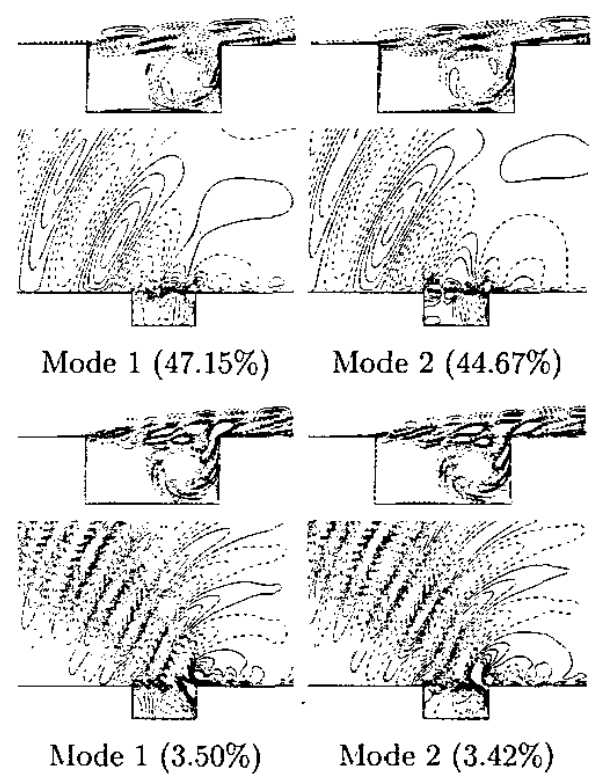

Figure 1: Vorticity (top) and dilatation (bottom) for vector-valued POD modes (snapshots from fully developed flow). Negative contours are dashed.

The 10-mode model actually blows up, and the 20mode model looks qualitatively very different for long time, with a complicated, chaotic-looking waveform. By contrast, the simulations based on vector-valued modes are qualitatively accurate even for long times. The 4-mode case drifts to a larger amplitude for long time, and the 6-mode and 11-mode cases are closer. This is in sharp contrast to the scalar mode case, where taking more modes made the dynamics worse for long time. A plausible reason why the vector-valued modes do better than scalar modes is the following: certain terms in the equations, namely the dilatation $u_{x}+v_{y}$, are sensitive, in that they involve a delicate cancellation between two large numbers (even though $u_{x}$ and $v_{y}$ are not small, the dilatation $u_{x}+v_{y}$ always remains small). If scalar-valued modes are used, $u$ and $v$ may drift apart slightly after some time, so that they no longer cancel each other, and the resulting error in dilatation can drive the equations in a non-physical way. We have observed this effect directly in Galerkin simulations from scalar-valued POD modes. When vector-valued modes are used, the dilatation is computed much more accurately, as it is effectively computed for each POD mode, and not from a sum of POD modes for different variables which may drift apart.

In order to use these models for control analysis or synthesis, it is of course necessary to introduce the effects of parametric variation and actuation into the models. 

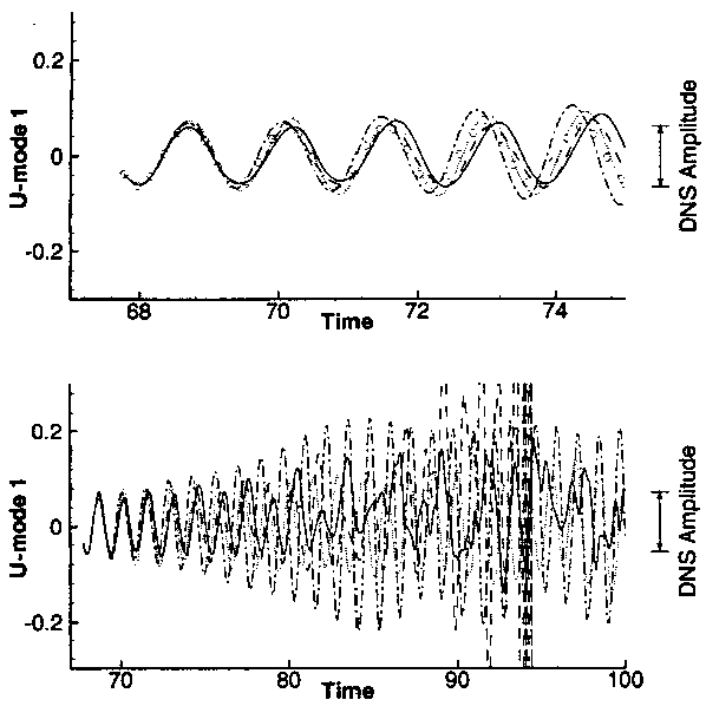

Scalar-valued modes
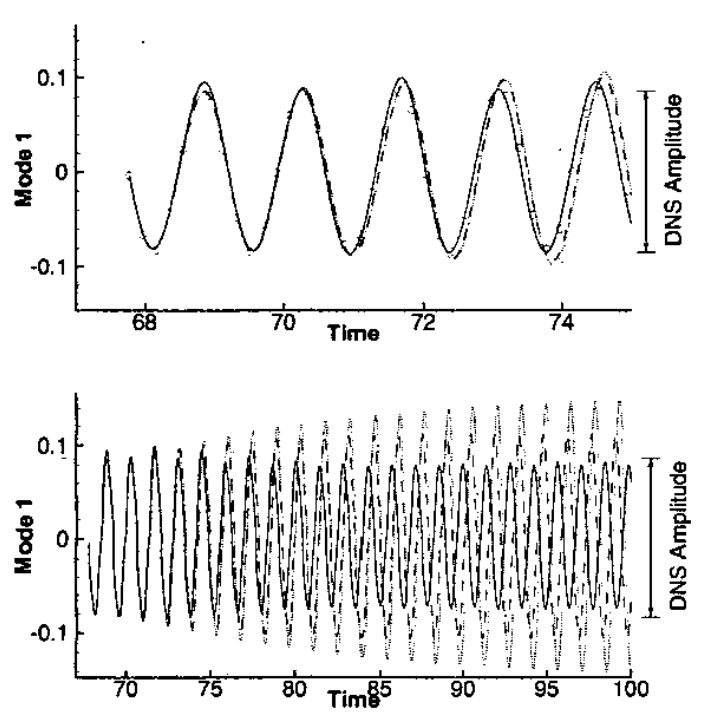

Vector-valued modes

Figure 2: Behavior of reduced-order models. For each model, two plots show the same simulation over different time intervals. Scalar POD modes: coefficient of $u$-velocity mode 1 , for projection of DNS (o), and POD/Galerkin models with 2 modes $(\cdots \cdots .),$.4 modes $(-.-), 10$ modes (-- ), and 20 modes (- - . Vector POD modes: coefficient of mode 1 , for projection of DNS (o), and POD/Galerkin models with 4 modes $(\cdots \cdots), 6$ modes (----), and 11 modes $(-)$.
Some efforts for the former will be presented in the full paper. For the cavity flow, it turns out the most of the parameters may be scaled out, and initial attempts at finding "universal" modes show some promise. The presence of an actuator will presumably change the flow structures, so POD modes need to be taken in the actuated flow, perhaps stacking snapshots from different runs. Precisely how to do this remains an open question, and is a topic for future research, but the present results are promising: since the unactuated flow can be accurately modeled by as few as 3 POD modes, it is likely the actuated flow may also be described by a model of very low dimension.

\section{POD modes in turbulent Jets}

In this example we consider a fully turbulent jet with Mach number 0.9 and Reynolds number 3600 that was computed with DNS in a previous study[5]. We compute POD modes for a variety of norms, including turbulent kinetic energy $(\alpha=(1,1,1,0,0))$, streamwise velocity, $(\alpha=(1,0,0,0,0))$, and pressure $(\alpha=$ $(0,0,0,0,1))$. We define norms over both individual planes normal to the jet axis (to compare with previous experiments[3]), the entire volume of the DNS, and on the surface of a large sphere in the acoustic field.

We discuss here some preliminary results relating to the structure of the POD modes, computed with various norms, and the convergence with which the POD modes reconstruct the acoustic far-field.

For most norms we consider, we find a significant amount of energy in relatively few POD modes. The norms based on surface-integrals converge more rapidly, in general, requiring 100 modes to capture $85 \%$ of the energy, for the case of streamwise velocity at a position near the end of the potential core. By contrast, 500 three-dimensional modes are required to capture the volume integral of pressure or kinetic energy to around $80 \%$.

For the pressure norm, we find in general that the most energetic POD modes have a wave packet structure, and that pairs of such modes represent advection of the structures at nearly constant phase speed, as was also the case for the cavity modes described in the previous section. One of each pair of the 8 most energetic pressure modes are shown in Figure 3, and corresponds to the axisymmetric and first helical Fourier modes in the azimuthal direction, as indicated on the plots. The most energetic modes bear a strong resemblance to the growth and decay of instability waves, and appear to coincide with the formation of vortices (both axisymmetric and helical). The third and forth most energetic modes (both axisymmetric and helical) shown a streamwise period doubling consistent with a vortex- 
pairing phenomena.

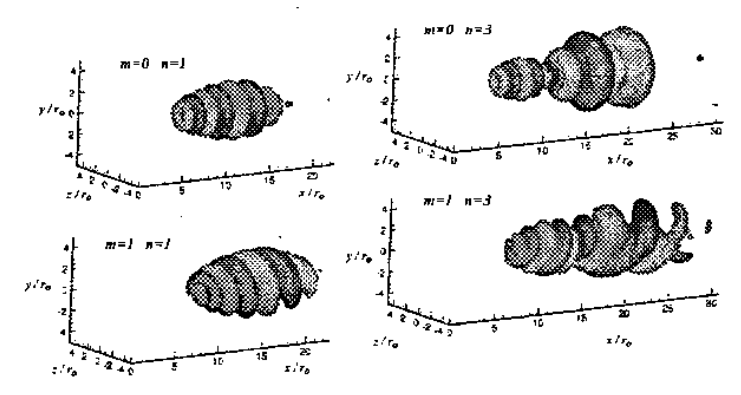

Figure 3: Most energetic POD modes (volume based norm of the pressure). In the labels, $m$ refers to the azimuthal mode number ( $m=0$ is the axisymmetric mode, $m=1$ is the helical mode) and $n$ refers to the POD mode number. The modes come in convecting pairs, with modes $n=2$ and $n=4$ resembling $n=1$ and $n=2$, respectively, bud phase shifted by 90 degrees.

The most energetic mode based on kinetic energy, by contrast, appears as a long, slowly rotating structure with azimuthal wavenumber two, and it is most intense past the end of the potential core. There is little discernable "propagation" of the kinetic energy modes.

We have examined the extent to which a portion of the POD modes are able to "reconstruct" various statistical quantities in the flow, such as (local) turbulent kinetic energy, Reynolds stresses, and pressure fluctuations both near and far (acoustic) field. For the pointwise near field quantities, the results are for the most part consistent with the rate at which the eigenvalues converge to the total (integrated) energy. One surprising result is that the pressure modes seem to have better overall pointwise convergence in the near field even for quantities that do not explicitly appear in the norm, such as turbulent kinetic energy.

In particular, we have found that all reconstructions based on norms defined over the near field give very poor convergence for the far acoustic field. While the basic directivity of the acoustic field is well represented by about 50 modes, it requires virtually all the modes to determine the sound pressure level to within one $\mathrm{dB}$. These results are shown in Figure 4. For reference, we also show reconstruction of the far field pressure based on pressure integrated on the surface of a large sphere in the far field. Only a few modes in that case capture the full acoustic field, and this indicates (not surprisingly) that the structure of the acoustic field is not particularly complex, only its relation with the near field dynamics.

We have also examined the structure of POD modes

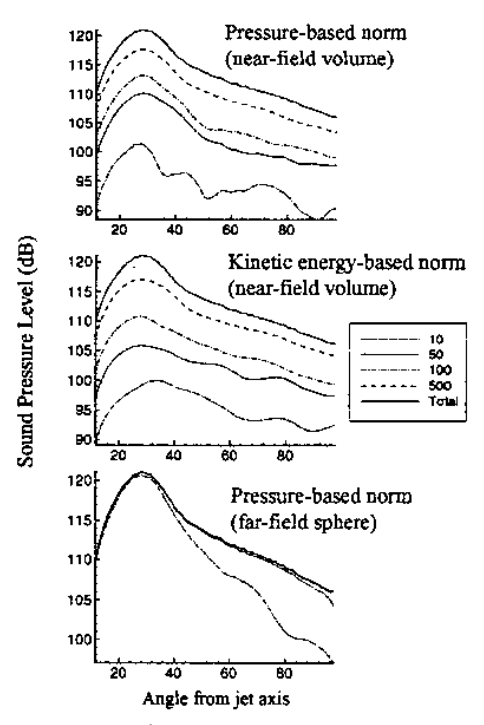

Figure 4: Reconstruction of the far field sound pressure level from POD modes defined with various norms.

(in the near-field) when the norm is based solely on the acoustic field (i.e. the large surface integral described above). The most energetic modes from this norm continue to display a strong wave packet structure in the near field. The wave packets seem to have an abrupt change in structure near the close of the potential core that may give rise to the acoustic radiation.

Further work is needed to further elucidate the connections between the POD modes, in various norms, and the sources of sound. Ultimately, our goal is to use that connection to develop reduced-order models, as noted above.

\section{Conclusions}

We have presented methods for applying POD and Galerkin projection to compressible flows, in the context of two models flows, namely self-sustained flow/acoustic oscillations in the flow over an open cavity, and a turbulent, transonic round jet.

For the jet, we have investigated the extent to which POD modes based on different norms lead to different energetic structures, and the extent to which near and far field statistics can be reconstructed with limited numbers of modes. While reconstruction of the far-field sound pressure level based on near field POD modes converges disappointingly slowly, our preliminary results show some interesting connections between the structure of the modes and the radiated acoustic field. In future, we intend to examine the connection 
between near and far-field modes to generate reducedorder models of sound generation by turbulent jets.

For the cavity, a much simpler transitional flow, the POD modes-especially vector-valued modes based on a enthalpy norm-were capable of describing the flow at very low order. Galerkin projections of (approximate) equations of motion onto these modes produced reduced-order models (with as few as 2 ODEs) which were able to predict the flow with quantitative accuracy for short time, and qualitatively for long time. It was shown why scalar-valued modes, where the intimate connections between the various flow variables are essentially lost, lead to reduced-order models with much more limited accuracy, and which are usually unstable for long times.

\section{References}

[1] R. E. A. Arndt, D. F. Long, and M. N. Glauser. The proper orthogonal decomposition of pressure fluctuations surrounding a turbulent jet. J. Fluid Mech., 340:1-33, 1997.

[2] L. N. Cattafesta, III, D. Shukla, S. Garg, and J. A. Ross. Development of an adaptive weapons-bay suppression system. AIAA Paper 99-1901, May 1999.

[3] J. H. Citriniti and W. K. George. Reconstruction of the global velocity field in the axisymmetric mixing layer utilizing the proper orthogonal decomposition. $J$. Fluid Mech., 418:137-166, 2000.

[4] T. Colonius. An overview of simulation, modeling, and active control of flow/acoustic resonance in open cavities. AIAA Paper 2001-0076, 2001.

[5] J. B. Freund. Noise sources in a low-Reynoldsnumber turbulent jet at Mach 0.9. J. Fluid Mech., 438:277-305, 2001.

[6] P. Holmes, J. L. Lumley, and G. Berkooz. Turbulence, Coherent Structures, Dynamical Systems and Symmetry. Cambridge University Press, Cambridge, 1996.

[7] L. Mongeau, H. Kook, and M. A. Franchek. Active control of flow-induced cavity resonance. AIAA/CEAS Paper 98-2349, 1998.

[8] J. E. Rossiter. Wind-tunnel experiments on the flow over rectangular cavities at subsonic and transonic speeds. Aeronautical Research Council Reports and Memoranda, No. 3438, October 1964.

[9] C. W. Rowley. Modeling, simulation and control of cavity flow oscillations. PhD thesis, California Institute of Technology, 2002.

[10] C. W. Rowley, T. Colonius, and A. Basu. On selfsustained oscillations in two-dimensional compressible flow over rectangular cavities. J. Fluid Mech., 455:315$346,2002$.
[11] Leonard Shaw and Stephen Northcraft. Closed loop active control for cavity acoustics. AIAA Paper 99-1902, May 1999.

[12] L. Sirovich. Chaotic dynamics of coherent structures. Parts I-III. Quarterly of Applied Math., XLV(3):561-582, 1987.

[13] M.J. Stanek, G. Raman, V. Kibens, J.A. Ross, J. Odedra, and J.W. Peto. Control of cavity resonance through very high frequency forcing. AIAA Paper 2000-1905, June 2000.

[14] D.R. Williams, D. Fabris, and J. Morrow. Experiments on controlling multiple acoustic modes in cavities. AIAA Paper 2000-1903, June 2000. 